\title{
Prediction of survival benefit when deciding between chemotherapy and best supportive therapy in elderly patients with advanced gastric cancer: A retrospective cohort study
}

\author{
AYA SUGIMOTO, TSUTOMU NISHIDA, NAOTO OSUGI, KEI TAKAHASHI, KAORI MUKAI, \\ DAI NAKAMATSU, TOKUHIRO MATSUBARA, SHIRO HAYASHI, MASASHI YAMAMOTO, \\ SACHIKO NAKAJIMA, KOJI FUKUI and MASAMI INADA \\ Department of Gastroenterology, Toyonaka Municipal Hospital, Toyonaka, Osaka 560-8565, Japan
}

Received July 18, 2018; Accepted November 13, 2018

DOI: $10.3892 / \mathrm{mco} .2018 .1772$

\begin{abstract}
It remains unclear whether elderly patients (EPs) with advanced gastric cancer (AGC) benefit from chemotherapy. The aim of the present study was to examine the prognostic factors for EPs with AGC in order to generate a prognosis-predicting scoring system. This single-center retrospective study examined consecutive patients with AGC between April 2012 and July 2017. Risk factors for survival in EPs aged $\geq 75$ years were identified using a Cox proportional hazards model, and a prognostic scoring system was generated and retrospectively evaluated to determine its usefulness for predicting patient prognosis. A total of 61 patients were enrolled as EPs (mean age, 81 years) and compared with 80 non-EPs (mean age, 66 years). The median survival time (MST) was significantly longer for non-EPs compared with that for EPs (3.8 vs. 10.1 months, respectively; $\mathrm{P}=0.0447)$. Among the $\mathrm{EPs}, 29$ (48\%) received chemotherapy and 32 received best supportive care (BSC). A total of 68 non-EPs $(85 \%)$ received chemotherapy and 12 non-EPs received BSC. Among EPs with AGC, age-adjusted multivariate analysis revealed that performance status (PS), neutrophil/lymphocyte ratio (NLR) $<4$, intestinal-type histology and chemotherapy were significant prognostic factors. To predict EPs too frail for chemotherapy prior to treatment, one point was assigned for a PS of 1, diffuse-type histology and NLR $\geq 4$, whereas 2 points were assigned for $\mathrm{PS} \geq 2$, and the point totals for each patient were calculated. A cut-off point of 2 had the best P-value by the log-rank test and was used to divide the patients into low-risk (LoR: Score 0-1) and high-risk (HiR: Score 2-4) groups. The MST of the LoR and HiR groups was 23.6 and 3.6 months,
\end{abstract}

Correspondence to: Dr Tsutomu Nishida, Department of Gastroenterology, Toyonaka Municipal Hospital, 4-14-1 Shibahara, Toyonaka, Osaka 560-8565, Japan

E-mail: tnishida.gastro@gmail.com

Key words: advanced gastric cancer, elderly patients, chemotherapy, best supportive care, prognostic factor respectively $(\mathrm{P}<0.001)$. As regards treatment strategies and risk groups, the LoR chemotherapy group had the best prognosis $(\mathrm{P}=0.0010)$, and LoR EPs who were administered chemotherapy had a longer MST (30.3 months) compared with EPs who received BSC (8.7 months). In conclusion, scoring systems using PS, histology and NLR may be useful when considering chemotherapy in EPs with AGC.

\section{Introduction}

Gastric cancer is the fifth most common type of cancer (1) and the third most common cause of cancer-related mortality worldwide (2). The majority of patients with gastric cancer are diagnosed between their late 60s and 80s. Consequently, the number of elderly patients (EPs) with advanced gastric cancer (AGC) has increased as a result of population aging and prolonged life expectancy; therefore, most AGC patients are elderly. Regarding treatment benefit for patients with AGC, a meta-analysis of overall survival (OS) in 35 clinical trials including a total of 5,726 patients revealed that chemotherapy significantly improved survival compared with best supportive care $(\mathrm{BSC})$ [hazard ratio $(\mathrm{HR})=0.37 ; 95 \%$ confidence interval (CI): 0.24-0.55] (3). However, EPs (aged $\geq 75$ years) often have multiple chronic medical conditions and develop adverse events induced by chemotherapy, as these individuals are frailer compared with younger patients with AGC. Consequently, EPs are usually excluded from the indications or are not considered to be suitable for chemotherapy to narrow the target when evaluating the efficacy and adverse events of chemotherapy in most clinical trials for AGC. Therefore, in practice, there is little evidence on whether EPs with AGC can tolerate chemotherapy and whether chemotherapy is beneficial in terms of survival.

The chemotherapy indications for EPs with AGC primarily depend on performance status (PS) and comorbidities. The attending physician subjectively decides upon the treatment strategy (chemotherapy vs. BSC) based on patient information rather than objective criteria. Therefore, it is difficult to distinguish between fit and frail EPs prior to treatment. The host's systemic inflammatory response (SIR) has recently emerged 
as a critical factor based on objective information on tumor progression (4). SIR includes common inflammation-based prognostic scores, such as C-reactive protein (CRP) (5); the Glasgow Prognostic Score (GPS) (6), which includes CRP and albumin; and the neutrophil/lymphocyte ratio (NLR) (7). Previous studies have revealed that SIR is associated with poor outcome in several types of cancer (8). However, whether SIR is useful for distinguishing between fit and frail EPs for chemotherapy remains unclear. Therefore, we hypothesized that scoring based on a combination of several SIR markers and other background characteristics on initial evaluation may predict the lifetime survival benefit for EPs with AGC, and help identify EPs who are likely to benefit from chemotherapy. In the present study, prognostic factors for EPs with AGC were examined to generate a prognostic scoring system to predict EPs who may be too frail for chemotherapy prior to treatment, and a subset from this scoring system was retrospectively evaluated to help predict prognosis or decide on treatment strategies.

\section{Patients and methods}

Patients. The present study was a single-center retrospective study including consecutive patients with AGC at Toyonaka Municipal Hospital (Toyonaka, Japan) between April 2012 and July 2017. During the study period, 173 patients diagnosed with AGC without surgical indication visited our department. Patients with surgically resectable disease were excluded and all patients enrolled had clinically confirmed evidence of metastatic lesions or unresectable factors. Patients who received chemotherapy were evaluated for the best response according to the Response Evaluation Criteria in Solid Tumors (version 1.1) and treatment toxicity according to the National Cancer Institute Common Terminology Criteria for Adverse Events (version 3.0). The present study was conducted in accordance with the principles outlined in the Declaration of Helsinki, and approval was obtained from the Institutional Review Board. This was a retrospective study involving human data that were previously collected and did not require the additional recruitment of human subjects; thus, the need for informed consent was waived via the opt-out method on our hospital website.

Treatment strategy and follow-up. The treatment strategy (chemotherapy or BSC) was decided by the attending physician according to the patient's background. The patients were routinely followed-up, including physical examination, laboratory testing, dynamic computed tomography scanning and esophagogastroduodenoscopy. If BSC was decided for a patient on initial evaluation, their physician was subsequently contacted to request follow-up information or investigate census registries. The latest follow-up was in August 2017. OS was calculated from the date of initial clinical diagnosis of AGC until death or the last available follow-up prior to death from any cause. Surviving patients were censored on their last follow-up date.

Data collection. The following factors were collected from medical records at the time of AGC diagnosis: Patient demographics [age, sex, Eastern Cooperative Oncology Group (ECOG) PS]; primary tumor locations; indications for chemotherapy; histological type; serum albumin level;
CRP; NLR; estimated glomerular filtration rate (eGFR); tumor markers [serum carcinoembryonic antigen (CEA) and carbohydrate antigen (CA) 19-9]; number of metastatic organs, including lymph nodes; and Charlson Comorbidity Index (9). Clinical course was also investigated, including chemotherapy agent and mean relative dose intensity (RDI), best response under chemotherapy, subsequent therapies and survival status. The RDI was defined as the ratio of the delivered dose intensity of each chemotherapy drug to the standard (referenced) dose intensity in the regimen. In the present study, RDI was evaluated during the planned first course of the first-line chemotherapy. The mean RDI was calculated as the mean percentage of RDI for all drugs in the first-line regimen.

Statistical analysis and prognostic scoring system. Median and range are reported for continuous variables. Categorical variables are summarized as frequency (percentage). Differences in variables were evaluated using the Wilcoxon signed-rank test or $\chi^{2}$ test. OS was estimated using the Kaplan-Meier method and compared using the log-rank test. HR and CI were estimated for OS.

Prognostic factors for survival in EPs were examined at the time of diagnosis of AGC via univariate and multivariate analyses using a Cox proportional hazards model. Subsequently, significant prognostic factors were identified and then clinical thresholds were set based on the best log-rank P-value. One point was assigned for each significant factor and the sum of the total points was calculated. Next, the best cut-off point to predict prognosis was evaluated. Finally, a prognostic scoring system was generated and the cut-off point was set.

All reported P-values are two-sided, and $\mathrm{P}<0.05$ is considered to indicate statistically significant differences. Statistical analyses were performed using JMP statistical software (version 13.1. 0, SAS Institute Inc., Cary, NC, USA).

\section{Results}

Patient characteristics. Of 173 patients with AGC, 143 had clinically confirmed evidence of unresectability or recurrence. Ultimately, 141 patients were enrolled in the present study, as 2 patients were treated at another hospital after diagnosis. The patient characteristics are summarized in Table I. S-1 plus cisplatin has been recommended as a standard treatment for AGC in Japan (10) based on the SPIRITS trial (11). The SPIRITS trial, however, was conducted with patients aged $<75$ years. For that reason, to evaluate the benefit of chemotherapy in EPs with AGC aged $\geq 75$ years, they were compared with non-EPs aged $<75$ years during the same study period. A total of 61 EPs [39 men; mean age, 81 years (range, 75-93 years)] and 80 non-EPs [59 men; mean age, 66 years (range, 36-74 years)] as a control group were enrolled in the present study. Of those patients, 29 EPs $(48 \%)$ and 68 non-EPs $(85 \%)$ received chemotherapy, whereas 32 EPs and 12 non-EPs received BSC based on the judgement of their attending physician. The reasons for BSC included the patient's wish $(n=12)$, poor PS $(n=9)$, advanced age $(n=6)$, dementia $(n=2)$ and others $(n=3)$ for Eps, and poor PS $(n=9)$, patient's wish $(n=1)$ and others $(n=2)$ for non-EPs.

A total of 53 EPs (87\%) and 73 non-EPs (91\%) had a target lesion. The median follow-up period was 187 days (range, 8-2,728 days). During the follow-up period, there were a total 
Table I. Characteristics of elderly patients and non-elderly patients with advanced gastric cancer.

\begin{tabular}{|c|c|c|c|c|c|c|c|c|}
\hline \multirow[b]{2}{*}{ Characteristics } & \multicolumn{4}{|c|}{ Elderly patients, total no. } & \multicolumn{4}{|c|}{ Non-elderly patients, total no. } \\
\hline & All, 61 & CT, 29 & BSC, 32 & P-value & All, 80 & $\mathrm{CT}, 68$ & BSC, 12 & P-value \\
\hline Male sex, no. $(\%)$ & $39(64)$ & $21(72)$ & $18(56)$ & 0.1892 & $59(74)$ & $49(72)$ & $10(83)$ & 0.4131 \\
\hline Median age, years (range) & $81(75-93)$ & 80 & 85.5 & 0.0003 & $66(36-74)$ & 66 & 68 & 0.2271 \\
\hline Median BMI, $\mathrm{kg} / \mathrm{m}^{2}$ (range) & $20.3(14-32)$ & 21.2 & 20.2 & 0.5203 & $20.5(14.2-30.5)$ & 20.3 & 23.4 & 0.1179 \\
\hline Diabetes mellitus, no. (\%) & $10(16)$ & $2(6.9)$ & $8(25)$ & 0.0565 & $15(19)$ & $13(19)$ & $2(17)$ & 0.8410 \\
\hline Hypertension, no. (\%) & $34(56)$ & $19(66)$ & $15(47)$ & 0.1432 & $22(28)$ & $17(25)$ & $5(42)$ & 0.2332 \\
\hline Dementia, no. (\%) & $6(9.8)$ & $0(0)$ & $6(19)$ & 0.0141 & $3(3.8)$ & $2(2.9)$ & $1(8.3)$ & 0.3647 \\
\hline Chronic kidney disease, no. (\%) & $48(79)$ & $21(72)$ & $27(84)$ & 0.2546 & $16(20)$ & $15(22)$ & $1(8.3)$ & 0.2731 \\
\hline Charlson Comorbidity Index ${ }^{a}$ & 0 & 0 & 1 & 0.0218 & 0 & 0 & 1 & 0.1156 \\
\hline Low/medium/high + very high $^{\mathrm{b}}$ & $37 / 22 / 2$ & $22 / 7 / 0$ & $15 / 15 / 2$ & 0.0473 & $52 / 24 / 4 / 0$ & $47 / 18 / 3 / 0$ & $5 / 6 / 1 / 0$ & 0.1845 \\
\hline PS $0-1$, no. (\%) & $46(75)$ & $26(900$ & $20(63)$ & 0.0324 & $63(79)$ & $62(91)$ & $1(8.3)$ & $<0.0001$ \\
\hline Histology type, intestinal, no. (\%) & $25(41)$ & $14(48)$ & $11(34)$ & 0.2703 & $25(31)$ & $21(31)$ & $4(33)$ & 0.8659 \\
\hline Gastric cardia tumor site, no. (\%) & $12(20)$ & $8(28)$ & $4(13)$ & 0.2296 & $17(21)$ & $16(24)$ & $1(8.3)$ & 0.2355 \\
\hline Target lesion, no. (\%) & $53(87)$ & $26(90)$ & $27(84)$ & 0.5418 & $73(91)$ & $65(96)$ & $8(67)$ & 0.00118 \\
\hline No. of metastatic lesions $\leq 1$, no. $(\%)$ & $25(41)$ & $14(48)$ & $11(34)$ & 0.2703 & $27(34)$ & $21(31)$ & $6(50)$ & 0.1966 \\
\hline CEA, median (range) & $\begin{array}{c}7.7 \\
(1-7446)\end{array}$ & 11.2 & 6.9 & 0.2692 & $\begin{array}{c}4.4 \\
(0.7-1737)\end{array}$ & 5.1 & 2.9 & 0.4846 \\
\hline CA19-9, median (range) & $\begin{array}{c}22 \\
(2-205307)\end{array}$ & 10 & 95.5 & 0.0691 & $\begin{array}{c}21.5 \\
(2-267426)\end{array}$ & 24 & 15 & 0.4952 \\
\hline Albumin $\geq 3 \mathrm{~g} / \mathrm{dl}$, no. (\%) & $34(56)$ & $21(72)$ & $13(41)$ & 0.0126 & $56(74)$ & $52(79)$ & $4(40)$ & 0.0094 \\
\hline CRP level <1 mg/dl, no. (\%) & $31(52)$ & $18(62)$ & $13(42)$ & 0.1189 & $38(49)$ & $37(55)$ & $1(9.1)$ & 0.0046 \\
\hline NLR $<4$, no. $(\%)$ & $29(48)$ & $17(61)$ & $12(38)$ & 0.0726 & $40(52)$ & $36(55)$ & $4(36)$ & 0.2638 \\
\hline
\end{tabular}

${ }^{a}$ The Charlson Comorbidity index encompasses 19 medical conditions weighted 1-6 with total scores ranging from 0 to $37 .{ }^{b}$ For each condition, the scores reflected the risk as follows: 0 , low; $1-2$, medium; $3-4$, high; and $\geq 5$, very high risk. CT, chemotherapy; BSC, best supportive care; CRP, C-reactive protein; eGFR, estimated glomerular filtration rate; NLR, neutrophil/lymphocyte ratio; PS, performance status; CEA, carcinoembryonic antigen; CA19-9, cancer antigen 19-9. Chronic kidney disease was defined as an eGFR $<60 \mathrm{ml} / \mathrm{min}$.
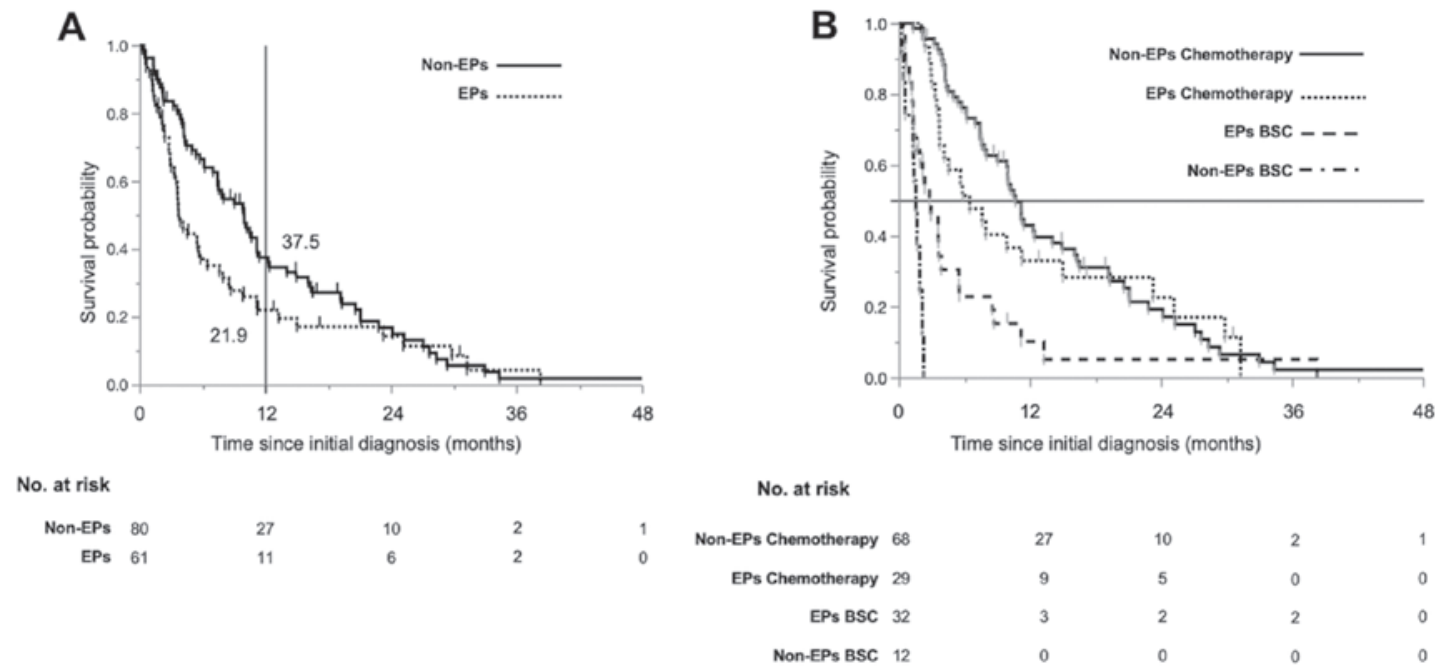

Figure 1. (A) OS in EPs and non-EPs with advanced gastric cancer. The 1-year OS rates in EPs and non-EPs were 21.9 and $37.5 \%$, respectively. A total of 51 EPs (84\%) and 68 non-EPs (85\%) died during the follow-up period. The cause of death was gastric cancer in 50 EPs (98\%) and 65 non-EPs (97\%). (B) OS of EPs as a function of treatment strategy and age. OS, overall survival; EPs, elderly patients; BSC, best supportive care.

of 119 deaths. The causes of death included cancer $[n=50$ (chemotherapy, $\mathrm{n}=23$ and BSC: $\mathrm{n}=27)]$ and treatment-related complications $(n=1)$ for EPs, and cancer $[n=65$ (chemotherapy, $\mathrm{n}=55$ and $\mathrm{BSC}, \mathrm{n}=10)$, treatment-related complications $(\mathrm{n}=2)$ and other causes $[\mathrm{n}=1$ (chemotherapy, $\mathrm{n}=1$ and $\mathrm{BSC}, \mathrm{n}=0)$ ] for non-EPs. 
Table II. Chemotherapy regimens and responses of patients with advanced gastric cancer.

\begin{tabular}{|c|c|c|c|}
\hline Items & EPs, $n=29$ & Non-EPs, $n=68$ & P-value \\
\hline \multicolumn{4}{|l|}{ First-line chemotherapy regimen, n (\%) } \\
\hline $\mathrm{S}-1 \pm$ trastuzumab & $16(53)$ & $5(7.4)$ & \\
\hline $\mathrm{S}-1+\mathrm{CDDP} \pm$ trastuzumab & $11(37)$ & $41(60)$ & \\
\hline $\mathrm{S}-1+\mathrm{CDDP}+$ docetaxel & $0(0)$ & $2(2.9)$ & \\
\hline $\mathrm{S}-1+\mathrm{L}-\mathrm{OHP} \pm$ trastuzumab or ramucirumab & $1(3.3)$ & $5(7.4)$ & \\
\hline $\mathrm{CAP}+$ trastuzumab & $0(0)$ & $1(1.5)$ & \\
\hline $\mathrm{CAP}+\mathrm{CDDP} \pm$ trastuzumab & $1(3.3)$ & $4(5.9)$ & \\
\hline $\mathrm{CAP}+\mathrm{CPT}-11+$ trastuzumab & $0(0)$ & $1(1.5)$ & \\
\hline Paclitaxel or nab-paclitaxel & $1(3.3)$ & $9(13)$ & \\
\hline Second-line chemotherapy, n (\%) & $7(24)$ & $46(68)$ & $<0.0001$ \\
\hline Treatment-related deaths, n (\%) & $1(3.3)$ & $2(2.9)$ & 1.000 \\
\hline \multicolumn{4}{|l|}{ Response to first-line chemotherapy, n (\%) } \\
\hline CR & $0(0)$ & $0(0)$ & 0.2464 \\
\hline PR & $5(17)$ & $14(21)$ & \\
\hline $\mathrm{SD}^{\mathrm{a}}$ & $10(34)$ & $34(50)$ & \\
\hline $\mathrm{PD}$ & $7(24)$ & $7(10)$ & \\
\hline $\mathrm{NE}$ & $7(24)$ & $13(19)$ & \\
\hline RR & $5(17)$ & $14(21)$ & 0.7866 \\
\hline DCR, n (\%) & $15(51)$ & $48(71)$ & 0.1035 \\
\hline Median average RDI, n (\%) & 90 & 100 & 0.0165 \\
\hline
\end{tabular}

ancluding non-CR/non-PD in case of patients without target lesions. CDDP, cisplatin; CAP, capecitabine; L-OHP, oxaliplatin; CPT-11, irinotecan; CR, complete response; PR, partial response; $\mathrm{SD}$, stable disease; PD, progressive disease; NE, not evaluated; RR, response rate; DCR, disease control rate; RDI, relative dose intensity.

OS. The OS data for EPs and non-EPs are plotted in Fig. 1A. The median survival time (MST) of non-EPs was significantly longer compared with that of EPs (10.1 vs. 3.8 months, respectively; $\mathrm{P}=0.0447)$; however, longer survival curves appeared to overlap 2 years after the initial diagnosis (Fig. 1A).

The survival curves grouped by age, with or without chemotherapy, are shown in Fig. 1B. There was a highly significant difference in OS between chemotherapy and BSC in non-EPs (10.8 vs. 1.6 months, respectively; $\mathrm{P}<0.0001)$, as well as in EPs (6.6 vs. 2.8 months, respectively; $\mathrm{P}=0.0069$ ). There was, however, a smaller lifetime survival benefit in EPs compared with that in non-EPs.

Efficacy, toxicity and feasibility of chemotherapy in EPs with $A G C$. The details of the chemotherapy regimen are shown in Table II. Among EPs who were administered chemotherapy, the response rate (RR) was $17 \%$, which was similar to that of non-EPs (21\%). The disease control rate (DCR) was lower in EPs compared with that in non-EPs (51 vs. 71\%, respectively), but this difference was not statistically significant. The median average RDI in EPs was 90\% (mean average RDI: 80\%). Among EPs treated with chemotherapy, second-line treatment was administered to 7 patients, which was a significantly lower percentage compared with that among non-EPs (24 vs. $68 \%$, respectively; $\mathrm{P}<0.0001)$.

Among patients who received chemotherapy, all toxicity grades were observed in 22 patients $(81 \%)$, and grade $>3$ toxicity was observed in 6 patients $(22 \%)$ (Table III). Of those patients, 2 continued the same regimen after dose reduction, but the remaining patients discontinued the first chemotherapy treatment as a result of grade 4 neutropenia or grade 3 fatigue, acneiform rash, palmar-plantar erythrodysesthesia syndrome or nausea. Finally, 6 patients $(22 \%)$ discontinued the first chemotherapy treatment as a result of treatment failure; 1 patient succumbed to febrile neutropenia.

Prognostic factors and scoring system in EPs with AGC. The mean CRP level in EPs was $2.5 \pm 3.4 \mathrm{mg} / \mathrm{dl}$, with a median of $0.69 \mathrm{mg} / \mathrm{dl}$ (range, $0-13.7 \mathrm{mg} / \mathrm{dl}$ ). The log-rank $\mathrm{P}$-values calculated for the CRP level were $\mathrm{P}=0.3053$ ( $<0.5$ vs. $\geq 0.5 \mathrm{mg} / \mathrm{dl}), \mathrm{P}=0.3014(<1 \mathrm{vs} . \geq 1 \mathrm{mg} / \mathrm{dl})$ and $\mathrm{P}=0.9347$ ( $<1.5 \mathrm{vs} . \geq 1.5 \mathrm{mg} / \mathrm{dl}$ ). The mean albumin level in EPs was $3.0 \pm 0.6 \mathrm{~g} / \mathrm{dl}$, with a median of $3 \mathrm{~g} / \mathrm{dl}$ (range, 1.5-4.1 g/dl). The log-rank P-values calculated for albumin were $\mathrm{P}=0.5548$ ( $<2.5$ vs. $\geq 2.5 \mathrm{~g} / \mathrm{dl}), \mathrm{P}=0.040(<3$ vs. $\geq 3 \mathrm{~g} / \mathrm{dl}$ ) and $\mathrm{P}=0.5662$ ( $<3.5 \mathrm{vs} . \geq 3.5 \mathrm{~g} / \mathrm{dl}$ ). The mean NLR in EPs was $7.4 \pm 8.5$, with a median of 4.3 (range, 1.2-46.5). The log-rank P-values calculated for NLR were $\mathrm{P}=0.2447$ ( $<3.5$ vs. $\geq 3.5$ ), $\mathrm{P}=0.0374$ ( $<4$ vs. $\geq 4)$ and $\mathrm{P}=0.1586(<4.5$ vs. $\geq 4.5)$. Based on the cut-off P-values for CRP, albumin and NLR, optimal cut-off levels of $1 \mathrm{mg} / \mathrm{dl}, 3.0 \mathrm{~g} / \mathrm{dl}$ and 4 , respectively, were determined. Subsequently, a univariate analysis was performed to identify significant prognostic factors. The results revealed that the following factors were significantly associated with better prognosis (Table IV): PS 0 or 1 , intestinal-type histology, albumin level $\geq 3 \mathrm{~g} / \mathrm{dl}$, CRP level $<1 \mathrm{mg} / \mathrm{dl}, \mathrm{NLR}<4$, 
Table III. Toxicities in elderly patients with advanced gastric cancer receiving chemotherapy.

\begin{tabular}{|c|c|c|c|}
\hline Toxicities & All grades & Grade 3 or 4 & Grade 5 \\
\hline \multicolumn{4}{|l|}{ Hematological, n (\%) } \\
\hline White blood cell decrease & $5(17)$ & $3(10)$ & 0 \\
\hline Neutropenia & $4(14)$ & $3(10)$ & 0 \\
\hline Anemia & $2(7.0)$ & 0 & 0 \\
\hline Thrombocytopenia & $1(3.4)$ & 0 & 0 \\
\hline Febrile neutropenia & $1(3.4)$ & 0 & $1(3.4)$ \\
\hline \multicolumn{4}{|l|}{ Non-hematological, n (\%) } \\
\hline Malaise & $12(41)$ & $1(3.4)$ & 0 \\
\hline Nausea & $9(31)$ & $1(3.4)$ & 0 \\
\hline Diarrhea & $4(14)$ & 0 & 0 \\
\hline Acute kidney injury & $1(3.4)$ & 0 & 0 \\
\hline Palmar-plantar erythrodysesthesia syndrome & $1(3.4)$ & $1(3.4)$ & 0 \\
\hline Peripheral sensory neuropathy & $1(3.4)$ & 0 & 0 \\
\hline Acneiform rash & $3(10)$ & $1(3.4)$ & 0 \\
\hline Skin hyperpigmentation & $1(3.4)$ & 0 & 0 \\
\hline Acute coronary syndrome & $1(3.4)$ & 0 & 0 \\
\hline
\end{tabular}


Figure 2. OS in EPs by total score according to the scoring system. (B) OS in EPs in the HiR and LoR groups as a function of treatment strategy. HiR, high-risk group; LoR, low-risk group. EPs, elderly patients; BSC, best supportive care; OS, overall survival.

number of metastatic lesions $\leq 1$ and treatment strategy. The age-adjusted multivariate analysis using these 7 significant factors revealed that PS $0(\mathrm{HR}=0.27,95 \% \mathrm{CI}: 0.10-0.75$, $\mathrm{P}=0.0113)$, PS $1(\mathrm{HR}=0.42,95 \% \mathrm{CI}: 0.17-0.797, \mathrm{P}=0.0274)$, intestinal-type histology $(\mathrm{HR}=0.41,95 \% \mathrm{CI}: 0.22-0.77$, $\mathrm{P}=0.0053), \mathrm{NLR}<4(\mathrm{HR}=0.35,95 \%$ CI: 0.14-0.79, $\mathrm{P}=0.0109)$ and treatment strategy $(\mathrm{HR}=0.42,95 \% \mathrm{CI}: 0.19-0.92$, $\mathrm{P}=0.0313$ ) were significant prognostic factors (Table IV). To predict EPs too frail for chemotherapy prior to treatment, 1 point was assigned for PS of 1, diffuse-type histology and $\mathrm{NLR} \geq 4$, and 2 points for $\mathrm{PS} \geq 2$, except for treatment strategy (Table V), and the sum of the total points for each patient was calculated as the score. Subsequently, 6 patients $(10 \%)$ with a total score of 0,10 patients $(17 \%)$ with a score of 1 , 22 patients $(37 \%)$ with a score of 2,15 patients $(25 \%)$ with a score of 3 and 4 patients $(12 \%)$ with a score of 4 were identified (Table IV). The survival curves for each score were well-separated (Fig. 2A). The mean total score among EPs was $2.1 \pm 1.1$ (median, 2). The log-rank P-values calculated for the total score were $\mathrm{P}=0.0016$ ( 0 vs. $\geq 1, \mathrm{HR}=0.21), \mathrm{P}<0.0001$ (0-1 vs. $2-4, \mathrm{HR}=0.21)$ and $\mathrm{P}<0.0001$ ( $0-2$ vs. $3-4, \mathrm{HR}=0.23)$. Therefore, a cut-off point of 2 was set (total score 0-1 vs. 2-4). EPs with a total score of 2-4 were classified as the high-risk (HiR) group ( $n=44$; chemotherapy, $n=17$ and BSC, $n=27$ ), and the remaining patients were classified as the low-risk (LoR) group ( $n=16$; chemotherapy, $n=11$ and $B S C, n=5)$. Among 
Table IV. Prognostic factors for overall survival in elderly patients with advanced gastric cancer based on the univariate analysis and on the multivariate analysis adjusted by age.

\begin{tabular}{|c|c|c|c|c|c|c|}
\hline \multirow[b]{2}{*}{ Factors } & \multicolumn{3}{|c|}{ Univariate analysis } & \multicolumn{3}{|c|}{ Multivariate analysis adjusted by age } \\
\hline & HR & $95 \%$ CI & P-value & HR & $95 \% \mathrm{CI}$ & P-value \\
\hline \multicolumn{7}{|l|}{ Age, years } \\
\hline$<80$ & 0.65 & $0.34-1.18$ & 0.1588 & & & \\
\hline$\geq 80$ & 1 & & & & & \\
\hline \multicolumn{7}{|l|}{ Sex } \\
\hline Male & 0.80 & $0.44-1.49$ & 0.4667 & & & \\
\hline Female & 1 & & & & & \\
\hline \multicolumn{7}{|l|}{ Performance status } \\
\hline 0 & 0.13 & $0.05-0.32$ & $<0.0001$ & 0.27 & $0.10-0.75$ & 0.0113 \\
\hline 1 & 0.37 & $0.15-0.62$ & 0.0015 & 0.42 & $0.17-0.97$ & 0.0274 \\
\hline$\geq 2$ & 1 & & & 1 & & \\
\hline \multicolumn{7}{|l|}{ CCI } \\
\hline High/very high & 1 & & & & & \\
\hline Medium & 1.00 & $0.20-18.2$ & 0.9967 & & & \\
\hline Low & 0.98 & $0.21-17.5$ & 0.9819 & & & \\
\hline \multicolumn{7}{|l|}{ Histology } \\
\hline Intestinal type & 0.56 & $0.31-0.99$ & 0.0490 & 0.41 & $0.22-0.77$ & 0.0053 \\
\hline Diffuse or other & 1 & & & 1 & & \\
\hline \multicolumn{7}{|l|}{ Albumin level, g/dl } \\
\hline$\geq 3$ & 0.38 & $0.21-0.68$ & 0.0012 & 1.00 & $0.45-2.20$ & 0.9942 \\
\hline$<3$ & 1 & & & 1 & & \\
\hline \multicolumn{7}{|l|}{ CRP level, mg/dl } \\
\hline$<1$ & 0.28 & $0.15-0.52$ & $<0.0001$ & 0.48 & $0.20-1.12$ & 0.0917 \\
\hline$\geq 1$ & 1 & & & 1 & & \\
\hline \multicolumn{7}{|l|}{ eGFR } \\
\hline$<60$ & 0.83 & $0.44-1.71$ & 0.5918 & & & \\
\hline$\geq 60$ & 1 & & & & & \\
\hline \multicolumn{7}{|l|}{ NLR } \\
\hline$<4$ & 0.25 & $0.13-0.48$ & $<0.0001$ & 0.35 & $0.14-0.79$ & 0.0109 \\
\hline$\geq 4$ & 1 & & & 1 & & \\
\hline \multicolumn{7}{|l|}{ Metastatic lesions } \\
\hline $0-1$ & 0.47 & $0.125-0.85$ & 0.0114 & 0.65 & $0.31-1.30$ & 0.2222 \\
\hline$\geq 2$ & 1 & & & 1 & & \\
\hline \multicolumn{7}{|l|}{ Treatment strategy } \\
\hline Chemotherapy & 0.47 & $0.26-0.82$ & 0.0086 & 0.42 & $0.19-0.92$ & 0.0313 \\
\hline $\mathrm{BSC}$ & 1 & & & 1 & & \\
\hline
\end{tabular}

CI, confidence interval; HR, hazard ratio; BSC, best supportive care; CRP, C-reactive protein; eGFR, estimated glomerular filtration rate; NLR, neutrophil/lymphocyte ratio; CCI, Charlson Comorbidity Index.

EPs, a score of 0-1 was a significantly good predictor of OS (age-adjusted HR for a score of 2-4: 0.21, 95\% CI: 0.09-0.44; $\mathrm{P}<0.0001)$. MST was significantly longer in the LoR compared with that in the HiR group (23.6 vs. 3.6 months, respectively; $\mathrm{P}<0.0001)$. Among the 4 groups based on treatment strategy and risk, the data revealed that LoR-chemotherapy had the best prognosis $(\mathrm{P}=0.0010)$ and that MST was longer in EPs treated with chemotherapy ( $\mathrm{n}=11$; MST, 30.3 months) compared with those treated with BSC in the LoR group ( $n=5$; MST, 8.7 months). The HiR-chemotherapy group had a significantly longer MST compared with the HiR-BSC group (4.2 vs. 2.4 months, respectively; $\mathrm{P}=0.0322$; Fig. $2 \mathrm{~B}$ ).

Similarly, non-EPs with a total score of 2-4 were classified as the HiR group ( $n=57$; chemotherapy, $n=46$ and BSC, $n=11$ ), and the remaining patients were classified as the LoR group $(n=20$; chemotherapy, $n=20$ and BSC, $n=0)$. The MST was significantly longer in the LoR compared with that in the HiR group (7.6 vs. 21.4 months, respectively; $P=0.0016$ ), which was similar to the results for non-EPs. In contrast to EPs, the HiR-chemotherapy non-EPs had a significantly longer MST 
Table V. Scoring indices for predicting survival in elderly patients with advanced gastric cancer.

\begin{tabular}{lcc}
\hline Scoring system & Score & \\
\hline Performance status & & \\
0 & 0 & \\
1 & 1 & \\
$\geq 2$ & & \\
Histology type & 0 & \\
Intestinal & 1 & \\
Diffuse & & \\
NLR & 0 & $2(2.6)$ \\
$<4$ & 1 & $18(23)$ \\
$\geq 4$ & EPs, $\mathrm{n}(\%)$ & Non-EPs, $(\%)$ \\
Total score (0-4) & $6(10)$ & $27(35)$ \\
0 & $10(17)$ & $9(12)$ \\
1 & $22(37)$ & 3 \\
2 & $15(25)$ & $20(26)$ \\
3 & $7(12)$ & $57(74)$ \\
4 & 1 & \\
Data missing & $16(27)$ & \\
Total score 0-1, low risk & $44(77)$ & \\
Total score 2-4, high risk & & \\
\hline
\end{tabular}

EPs, elderly patients; non-EPs, non-elderly patients; NLR, neutrophil/lymphocyte ratio.

compared with the HiR-BSC non-EPs (8.1 vs. 1.7 months, respectively; $\mathrm{P}<0.0001)$.

\section{Discussion}

The populations of industrial countries, including Japan, are aging rapidly. Although $>60 \%$ of patients with newly developed cancer and $>70 \%$ of cancer fatalities are reported in elderly populations in the United States and Europe (12), EPs are generally under-represented in clinical trials $(13,14)$. Two retrospective analyses of clinical trials involving patients with solid tumors suggested that older age does not necessarily diminish the tolerance or response to chemotherapy (15); however, there is little evidence on the benefit of chemotherapy for EPs with cancer.

The majority of patients with gastric cancer are diagnosed between their late 60s and 80s. The SPIRITS trial showed an advantage for S-1, an oral FU antitumor drug, combined with cisplatin for the first-line treatment of AGC (11). Consequently, S-1 plus cisplatin was recommended as a standard treatment for AGC in Japan (10), although S-1 plus cisplatin was not found to be superior to 5-FU plus cisplatin in a global randomized trial conducted outside Japan (FLAGS) (16). The SPIRITS trial, however, included patients aged $<75$ years. In previous phase II studies, Koizumi et al (17) and Imamura et al (18) demonstrated that S-1 monotherapy was safe and efficacious for EPs with AGC. These two trials were conducted with 33 and 35 AGC patients, respectively, aged $>75$ years, and they reported
RRs of 21 and 14\%, respectively, and a median OS of 15.7 and 14.6 months, respectively. Regarding adverse events, anemia and anorexia were predominantly observed, but no treatment-related deaths occurred. In the SPIRITS trial, the median OS in younger patients receiving S-1 monotherapy was 11.0 months, which was shorter compared with that in the abovementioned studies in EPs. This finding reflects the enrollment of only selected EPs who were considered fit for chemotherapy in both studies.

Several recent studies have demonstrated that SIR is associated with prognosis in various types of cancer $(5,6,8)$. Previous studies have reported that albumin, CRP and NLR are important prognostic factors in patients with gastric cancer and other types of cancer (19-21). In the present study, we proposed a scoring system using 3 factors, namely PS, histological type and NLR, identified as significant in a multivariate analysis including SIR. This scoring system separated EPs who may benefit more from chemotherapy from those who may benefit less. In brief, MST was longer in EPs treated with chemotherapy in the LoR compared with those treated with chemotherapy in the HiR group. However, a clinically relevant chemotherapy benefit was not observed for EPs in the HiR group, as survival with chemotherapy was only prolonged from 2.4 to 4.2 months (Fig. 2B). Based on these results, this scoring system may be valuable for selecting EPs fit for chemotherapy, and risk factors should be taken into consideration when determining the indications for chemotherapy for EPs with AGC.

Several studies have reported the clinicopathological characteristics of EPs with gastric cancer. Arai et al (22), reported that differentiated-type adenocarcinoma accounted for $50 \%$ of advanced cancers among 994 Japanese patients aged $\geq 65$ years. The proportion of differentiated-type carcinoma increased significantly with advancing age in female patients with advanced cancer, but no significant change was observed in male patients with advanced cancer. Other reports $(23,24)$ have also found an increased proportion of differentiated-type carcinoma with increasing age. Mizoe et al (25), reported higher 5- and 10-year survival rates in patients with differentiated-type gastric cancer compared with patients with the undifferentiated type; the authors hypothesized that this difference may be due to the rapid tumor growth of undifferentiated-type cancer.

The present study has several limitations due to its retrospective nature. First, the number of patients at a single institute was small, and the patient sample was divided into two groups using 75 years as the cut-off age, as most clinical trials have been conducted with patients aged $<75$ years. Consequently, the age for non-EPs ranged from 36 to 74 years, with a wider distribution compared with that for EPs. Second, the treatment strategy or regimen relied upon the physician's decision; however, a strength of the present study was the evaluation of prognostic factors in patients, including non-EPs or patients receiving BSC with poor PS, compared with those receiving chemotherapy in the real world, rather than in a clinical trial, during the same period in the same hospital. Moreover, the present study identified potential EPs who may benefit from chemotherapy. Third, a common cut-off value for SIR has not been established. Several cut-off values are used for CRP and NLR, and these values are not consistent, even for the same type of cancer $(26,27)$. In the present study, 
optimal cut-off levels were set for CRP $(1 \mathrm{mg} / \mathrm{dl})$, albumin $(3.0 \mathrm{~g} / \mathrm{dl})$ and NLR (4). Zhang et al (19), evaluated whether NLR was a useful prognostic factor in patients with gastric cancer using a meta-analysis; the results of a subgroup analysis of previous studies demonstrated that the prognostic value of NLR was not substantially affected by the cut-off value. In the present study, that NLR was found to be of significant prognostic value in EPs. It may also be useful to consider NLR when determining indications for chemotherapy in frail patients with AGC. Finally, the screening tools for EPs were not sufficiently evaluated, although no significant factors were found based on the Charlson Comorbidity Index. The optimal screening tools for EPs have not yet been established. However, Decoster et al (28) conducted a systematic review based on 17 different screening tools in EPs, and concluded that G8 was the best screening tool due to its high sensitivity with acceptable specificity and prognostic value for predicting outcome measures. In the present study, the G8 health status scores were not routinely evaluated during the study period. In the future, however, the scoring system must be verified in a validation cohort compared with G8. Additionally, we did not evaluate quality of life (QOL) in this elderly population. Although chemotherapy is often used to improve QOL for patients with end-stage cancer, slightly extending the MST with chemotherapy must be weighed against QOL in HiR EPs.

In conclusion, the lifetime survival benefit from chemotherapy was found to be greater in non-EPs compared with that in EPs with AGC. However, EPs with AGC who were placed in the LoR group by the scoring system had reasonable OS compared with non-EPs with AGC. Therefore, EPs with AGC in the HiR group should be evaluated individually and receive special attention in order to determine the indications for chemotherapy.

\section{Acknowledgements}

Not applicable.

\section{Funding}

No funding was received.

\section{Availability of data and materials}

The datasets used during the present study are available from the corresponding author upon reasonable request.

\section{Authors' contributions}

AS and TN collected and analyzed the data and wrote the manuscript. AS, TN, NO, KT, KM, DN, TM, SH, MY, SN, KF and MI provided medical care for the patients enrolled in this study.

\section{Ethics approval and consent to participate}

The present study was conducted in accordance with the Declaration of Helsinki, and approval was obtained from the Institutional Review Board of the Toyonaka Municipal Hospital (2015-10-06).

\section{Patient consent for publication}

This is a retrospective study involving human data that was previously collected and did not require the additional recruitment of human subjects; thus, the need for informed consent was waived via the opt-out method of our hospital website.

\section{Competing interests}

The authors declare that they have no competing interests.

\section{References}

1. International Agency for Research on Cancer: Estimated number of incident cases, both sexes, worldwide (top 10 cancer sites) in 2012. 2012; http://gco.iarc.fr/today/online-analysis-multi-bar $\mathrm{s} ?$ mode $=$ cancer $\&$ mode $\_$population $=$continents $\&$ populatio $\mathrm{n}=900 \& \operatorname{sex}=0 \&$ cancer $=29 \&$ type $=0 \&$ statistic $=0$ \& prevalence $=0 \&$ color_palette $=$ default. Accessed September 11, 2018 .

2. International Agency for Research on Cancer: Estimated number of deaths, both sexes, worldwide (top 10 cancer sites) in 2012 . 2012; http://gco.iarc.fr/today/online-analysis-multi-bars?mode $=\mathrm{c}$ ancer\&mode_population $=$ continents $\&$ population $=900 \&$ sex $=0 \&$ ca ncer $=29 \&$ type $=1 \&$ statistic $=0 \&$ prevalence $=0 \&$ color_palette $=$ default Accessed September 11, 2018.

3. Wagner AD, Unverzagt S, Grothe W, Kleber G, Grothey A, Haerting $J$ and Fleig WE: Chemotherapy for advanced gastric cancer. Cochrane Database Syst Rev 3: CD004064, 2010.

4. Hanahan D and Weinberg RA: Hallmarks of cancer: The next generation. Cell 144: 646-674, 2011.

5. Szkandera J, Stotz M, Absenger G, Stojakovic T, Samonigg H, Kornprat P, Schaberl-Moser R, Alzoughbi W, Lackner C, Ress AL, et al: Validation of C-reactive protein levels as a prognostic indicator for survival in a large cohort of pancreatic cancer patients. Br J Cancer 110: 183-188, 2014.

6. McMillan DC, Forrest LM, O'Gorman P, Angerson WJ and McArdle CS: Performance status of male and female advanced cancer patients is independently predicted by mid-upper arm circumference measurement. Nutr Cancer 42: 191-193, 2002.

7. Wei Y, Jiang YZ and Qian WH: Prognostic role of NLR in urinary cancers: A meta-analysis. PLoS One 9: e92079, 2014

8. Imrie CW: Host systemic inflammatory response influences outcome in pancreatic cancer. Pancreatology 15: 327-330, 2015.

9. Charlson ME, Pompei P, Ales KL and MacKenzie CR: A new method of classifying prognostic comorbidity in longitudinal studies: Development and validation. J Chronic Dis 40: 373-383, 1987.

10. Sano T and Aiko T: New Japanese classifications and treatment guidelines for gastric cancer: Revision concepts and major revised points. Gastric Cancer 14: 97-100, 2011.

11. Koizumi W, Narahara H, Hara T, Takagane A, Akiya T, Takagi M, Miyashita K, Nishizaki T, Kobayashi O, Takiyama W, et al: S-1 plus cisplatin versus S-1 alone for first-line treatment of advanced gastric cancer (SPIRITS trial): A phase III trial. Lancet Oncol 9: 215-221, 2008.

12. Aapro MS, Köhne CH, Cohen HJ and Extermann M: Never too old? Age should not be a barrier to enrollment in cancer clinical trials. Oncologist 10: 198-204, 2005.

13. Hutchins LF, Unger JM, Crowley JJ, Coltman CA Jr and Albain KS: Underrepresentation of patients 65 years of age or older in cancer-treatment trials. N Engl J Med 341: 2061-2067, 1999.

14. Monfardini S, Sorio R, Boes GH, Kaye S and Serraino D: Entry and evaluation of elderly patients in European Organization for Research and Treatment of Cancer (EORTC) new-drug-development studies. Cancer 76: 333-338, 1995.

15. Giovanazzi-Bannon S, Rademaker A, Lai G and Benson AB III: Treatment tolerance of elderly cancer patients entered onto phase II clinical trials: An Illinois Cancer Center study. J Clin Oncol 12: 2447-2452, 1994.

16. Ajani JA, Rodriguez W, Bodoky G, Moiseyenko V, Lichinitser M, Gorbunova V, Vynnychenko I, Garin A, Lang I and Falcon S: Multicenter phase III comparison of cisplatin/S-1 with cisplatin/infusional fluorouracil in advanced gastric or gastroesophageal adenocarcinoma study: The FLAGS trial. J Clin Oncol 28: 1547-1553, 2010. 
17. Koizumi W, Akiya T, Sato A, Sakuyama T, Sasaki E, Tomidokoro T, Hamada T, Fujimori M, Kikuchi Y, Shimada K, et al: Phase II study of S-1 as first-line treatment for elderly patients over 75 years of age with advanced gastric cancer: The Tokyo Cooperative Oncology Group study. Cancer Chemother Pharmacol 65: 1093-1099, 2010.

18. Imamura H, Kishimoto T, Takiuchi H, Kimura Y, Morimoto T, Imano M, Iijima S, Yamashita K, Maruyama K, Otsuji T, et al: Phase II study of S-1 monotherapy in patients over 75 years of age with advanced gastric cancer (OGSG0404). J Chemother 26: 57-61, 2014.

19. Zhang X, Zhang W and Feng LJ: Prognostic significance of neutrophil lymphocyte ratio in patients with gastric cancer: A meta-analysis. PLoS One 9: e111906, 2014

20. Hwang JE, Kim HN, Kim DE, Choi HJ, Jung SH, Shim HJ, Bae WK, Hwang EC, Cho SH and Chung IJ: Prognostic significance of a systemic inflammatory response in patients receiving first-line palliative chemotherapy for recurred or metastatic gastric cancer. BMC Cancer 11: 489, 2011.

21. Namikawa T, Munekage E, Munekage M, Maeda H, Yatabe T, Kitagawa H, Kobayashi $M$ and Hanazaki K: Evaluation of systemic inflammatory response biomarkers in patients receiving chemotherapy for unresectable and recurrent advanced gastric cancer. Oncology 90: 321-326, 2016.

22. Arai T, Esaki Y, Inoshita N, Sawabe M, Kasahara I, Kuroiwa K, Honma N and Takubo K: Pathologic characteristics of gastric cancer in the elderly: A retrospective study of 994 surgical patients. Gastric Cancer 7: 154-159, 2004.

23. Wang JY, Hsieh JS, Huang CJ, Huang YS and Huang TJ: Clinicopathologic study of advanced gastric cancer without serosal invasion in young and old patients. J Surg Oncol 63 . 36-40, 1996.
24. Hashimoto H, Esaki Y, Kino K, Takahashi T and Takubo K: Gastric cancer in the elderly - relationship between macroscopic appearance and histological type in autopsy specimens. Nippon Ronen Igakkai Zasshi 34: 499-505, 1997 (In Japanese).

25. Mizoe A, Tanaka K, Ura K, Izawa K, Tsunoda T and Kanematsu T: Long term prognosis according to histological type of gastric cancer. J Jpn Pract Surg Soc 54: 1732-1737, 1993 (In Japanese).

26. Forrest LM, McMillan DC, McArdle CS, Angerson WJ and Dunlop DJ: Evaluation of cumulative prognostic scores based on the systemic inflammatory response in patients with inoperable non-small-cell lung cancer. Br J Cancer 89: 1028-1030, 2003.

27. Osugi J, Muto S, Matsumura Y, Higuchi M, Suzuki H and Gotoh M: Prognostic impact of the high-sensitivity modified Glasgow prognostic score in patients with resectable non-small cell lung cancer. J Cancer Res Ther 12: 945-951, 2016.

28. Decoster L, Van Puyvelde K, Mohile S, Wedding U, Basso U, Colloca G, Rostoft S, Overcash J, Wildiers H, Steer C, et al: Screening tools for multidimensional health problems warranting a geriatric assessment in older cancer patients: An update on SIOG recommendations. Ann Oncol 26: 288-300, 2015.

This work is licensed under a Creative Commons Attribution-NonCommercial-NoDerivatives 4.0 International (CC BY-NC-ND 4.0) License. 